\title{
X-Ray Signatures of Axion Conversion in Magnetic White Dwarf Stars
}

\author{
Christopher Dessert, Andrew J. Long, and Benjamin R. Safdi \\ Leinweber Center for Theoretical Physics, University of Michigan, Ann Arbor, Michigan 48109, USA
}

(Received 25 March 2019; revised manuscript received 25 June 2019; published 7 August 2019)

\begin{abstract}
White dwarf (WD) stars may radiate keV-energy axions produced in their stellar cores. This has been extensively studied as an extra channel by which WDs may cool, with some analyses even suggesting that axions can help explain the observed WD luminosity function. We show that the radiated axions may convert into $\mathrm{x}$ rays in the strong magnetic fields surrounding the WDs, leading to observable $\mathrm{x}$-ray signatures. We use Suzaku observations of the WD RE J0317-853 to set the strongest constraints to date on the combination of the axion-electron $\left(g_{\text {aee }}\right)$ times axion-photon $\left(g_{a y \gamma}\right)$ couplings, and we show that dedicated observations of magnetic WDs by telescopes such as Chandra, XMM-Newton, and NuSTAR could increase the sensitivity to $\left|g_{\text {aee }} g_{\text {ayy }}\right|$ by over an order of magnitude, allowing for a definitive test of the axionlike-particle explanation of the stellar cooling anomalies.
\end{abstract}

DOI: 10.1103/PhysRevLett.123.061104

The quantum chromodynamics (QCD) axion, originally proposed to solve the strong $C P$ problem [1-4], is a wellmotivated extension of the standard model of particle physics. The QCD axion is a light pseudoscalar particle that couples to the QCD operator $G \tilde{G}$, with $G$ the QCD field strength. Additionally, the axion has dimension-5 couplings to electromagnetism and to matter. Studies of string compactifications show that, in addition to the QCD axion, there may exist a number of additional light pseudoscalar particles, with couplings to electromagnetism and matter but not to QCD $[5,6]$. These pseudoscalars are called axionlike-particles (ALPs), though throughout this work we will refer to all such particles as axions. In this work we present a novel method, using x-ray observations of magnetic white dwarf (WD) stars (MWDs), to probe the existence of axions.

WDs have long been used as probes of axions by studying the possibility of energy loss from axion emission [7]. Axions are emitted by axion bremsstrahlung in electron-nucleon scattering. The extra energy loss would modify WD cooling and thus change the luminosity function of WDs. Comparisons to the observed luminosity function have been used to set stringent constraints on the axion-electron coupling [8-10]. Moreover, it has been suggested that the observed WD luminosity function actually prefers the existence of an axion [8,9], a claim further supported by period-drift measurements of WDs undergoing pulsations [11].

Published by the American Physical Society under the terms of the Creative Commons Attribution 4.0 International license. Further distribution of this work must maintain attribution to the author(s) and the published article's title, journal citation, and DOI. Funded by SCOAP ${ }^{3}$.
In this work we propose to use $\mathrm{x}$-ray observations of MWDs to detect the small fraction of emitted axions that convert to x-ray photons outside of the MWD in the strong surrounding magnetic field, as illustrated in Fig. 1. The conversion of axions to photons utilizes the axion-photon coupling. The proposed method uses the following key properties of isolated MWDs. First, the surrounding magnetic fields can be quite high, $\sim 10^{9} \mathrm{G}[12,13]$, enhancing the axion-photon conversion probability. Second, while the core temperature of WDs is typically in the x-ray band, $T_{c} \sim 10^{7} \mathrm{~K}$, the effective surface temperature is significantly lower, $T_{\text {eff }} \sim 10^{4} \mathrm{~K}$ (see, e.g., Ref. [14]). Therefore, an isolated MWD should not produce $\mathrm{x}$ rays in the absence of axions. X-ray energy axions may escape the core and then convert into real $\mathrm{X}$-ray photons in the magnetic field surrounding the WD, leading to a nearly thermal x-ray flux

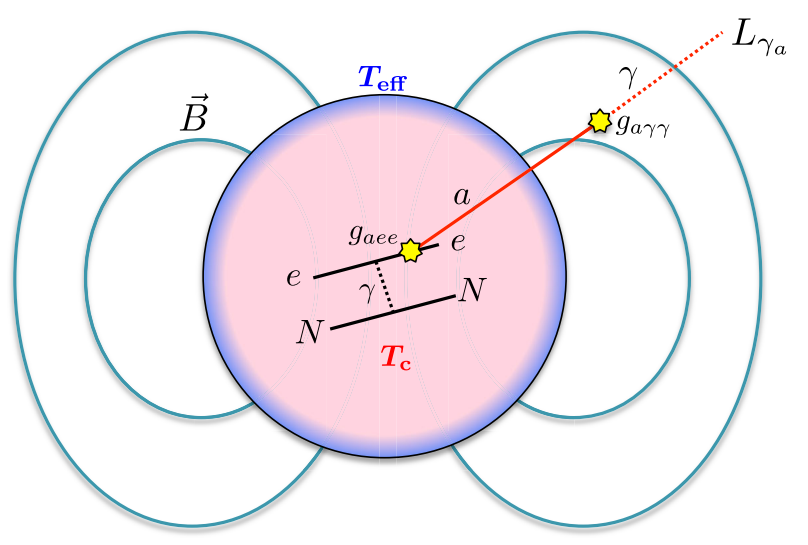

FIG. 1. Axions are produced inside of a WD star and convert into x-ray photons as they pass through the star's magnetic field. The axion-induced $\mathrm{x}$ rays have energy around the core temperature $T_{c}$, which is much higher than the WD surface temperature $T_{\text {eff }}$. 
at the temperature of the core. In this work, we show that $\mathrm{x}$-ray telescope observations of MWDs have the potential to probe a wide range of axion masses and couplings. However, as we will show, the x-ray observations are only sensitive to low-mass ALPs and not to the QCD axion, as the axion-photon conversion probability is suppressed for large axion masses.

A similar approach to axion detection was previously suggested for neutron stars (NSs) [15]. In that case, nuclear bremsstrahlung in the NS generates an outgoing flux of axions, which may convert into x rays in the strong magnetic field surrounding the NS. However, it was pointed out in Ref. [16] that vacuum birefringence effects from quantum electrodynamics (QED), in the presence of strong magnetic fields, stymie the axion-photon conversion process. The result is that the axion-induced x-ray flux from NSs is expected to be negligible for a Kim-ShifmanVainshtein-Sakharov [17-19] or Dine-Fischler-SrednickiZhitnitsky [20,21] QCD axion, but the flux may still be significant for ALPs $[22,23]$. We will discuss these QED effects for MWDs below.

In many ways our proposal is reminiscent of the CAST experiment [24-26], which looks for axions produced within the Sun. These keV-energy axions travel to Earth where they are converted into x-ray photons by the strong magnetic field of the CAST experiment and detected with $\mathrm{x}$-ray optics. The key conceptual difference between our MWD proposal and CAST, in addition to using MWDs as the axion source instead of the Sun, is that the conversion to $\mathrm{x}$ rays takes place not in the lab but rather in the magnetosphere surrounding the MWD itself. A similar approach has in fact been suggested for the Sun (see, e.g., Ref. [27]), whereby one looks at spectral and morphological distortions to the solar x-ray spectrum from axion-photon conversion in the solar magnetic field, but this is complicated by the fact that the Sun is already a strong x-ray source.

We note that a host of additional astrophysical probes of axions and axion dark matter (DM) have been proposed. These include radio signatures of axion DM conversion in NSs [28-31] and radio signatures of axion decay [32], supernova cooling [33,34], energy loss in horizontal branch stars in globular clusters [35], and photon-axion oscillations leading to increased transparency of $\mathrm{TeV}$ gamma rays [36-39]. Axion DM is also the subject of significant laboratory efforts at present [40-54].

$X$-ray flux calculation.- It is useful to recall how an axion interacts with matter (see Ref. [55] for a review). The QCD axion couples to gluons through the operator $\mathcal{L} \supset-a \alpha_{s} G \tilde{G} /\left(8 \pi f_{a}\right)$, where $a$ is the axion field, $\alpha_{s}$ is the strong fine structure constant, $f_{a}$ is the axion decay constant, and $G$ is the QCD field strength tensor. The axion may also couple to electromagnetism through the operator

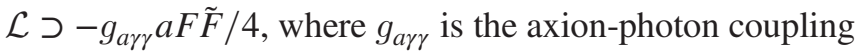
with units of $\mathrm{GeV}^{-1}$, and $F$ is the QED field strength tensor. Finally, the axion can interact with electrons through the operator $\mathcal{L} \supset g_{\text {aee }} /\left(2 m_{e}\right) \bar{e} \gamma^{\mu} \gamma_{5} e \partial_{\mu} a$, where $g_{\text {aee }}$ is the dimensionless axion-electron coupling, $m_{e}$ is the electron's mass, and $e$ is the electron field. It is customary to write $g_{a \gamma \gamma}=C_{\gamma} \alpha_{\mathrm{em}} /\left(2 \pi f_{a}\right)$ and $g_{a e e}=C_{e} m_{e} / f_{a}$, where $\alpha_{\mathrm{em}}$ is the electromagnetic fine structure constant and $C_{\gamma}$ and $C_{e}$ are dimensionless parameters.

The coupling of axions to matter allows axions to be emitted from WDs. WDs have dense cores that are supported by electron degeneracy pressure and consist predominantly of a hot plasma of carbon, oxygen, and electrons. The temperature of this isothermal plasma, which makes up the bulk of the matter of the WD, can be $T_{c} \approx 10^{7} \mathrm{~K}$ or higher, depending on the WD. Axions are emitted most efficiently in WDs from bremsstrahlung off of electrons in electron-nuclei scattering, through the diagram illustrated in Fig. 1. The luminosity radiated from a WD in axions, denoted by $L_{a}$, is calculated in Refs. [7,56,57] (see also Ref. [58]), and for a carbon oxygen WD with mass density ratio 0.5 and total mass $M_{\mathrm{WD}}$, we find

$$
\frac{L_{a}}{L_{\odot}} \approx 1.6 \times 10^{-4}\left(\frac{g_{a e e}}{10^{-13}}\right)^{2}\left(\frac{M_{\mathrm{WD}}}{1 M_{\odot}}\right)\left(\frac{T_{c}}{10^{7} \mathrm{~K}}\right)^{4},
$$

where $L_{\odot}$ and $M_{\odot}$ are the luminosity and mass of the Sun, respectively. The energy spectrum of the axion emission is found to be thermal at temperature $T_{c}: d L_{a} / d E \propto$ $E^{3} /\left(e^{E / T_{c}}-1\right)[56]$.

The emitted axions may be converted into x-ray photons in the magnetic field surrounding the MWD. Under the approximation where we assume all axions travel along radial trajectories originating from the MWD center, we may derive a simple analytic expression for the conversion probability. Note that in the Supplemental Material (SM) [59] we derive and numerically solve the relevant equations of motion for the more general trajectories and field configurations appropriate for a realistic MWD with finite extent, but the results are similar to those found by assuming radial trajectories. The axion-photon mixing equations in the presence of an external magnetic field can be reduced to a system of first-order differential equations using a WKB approximation. This approximation assumes that the scale of variation in the magnetic field is much larger than the axion's de Broglie wavelength. By working in the Weyl gauge, $A^{0}=0$, and focusing on axion trajectories along which the angle with respect to the magnetic field does not change, these equations take the form [16]

$$
\left[i \partial_{r}+E+\left(\begin{array}{cc}
\Delta_{\|} & \Delta_{B} \\
\Delta_{B} & \Delta_{a}
\end{array}\right)\right]\left(\begin{array}{c}
A_{\|} \\
a
\end{array}\right)=0,
$$

where $E$ is the axion's energy. Here $A_{\|}(r)$ denotes the vector potential component in the plane normal to the direction of propagation and parallel to the external magnetic field, as a function of the radial coordinate $r$, while $a(r)$ is the axion field. The probability for an axion to convert into a photon $p_{a \rightarrow \gamma}$ is determined by solving Eq. (2) and comparing the 
magnitude squared of an initial pure axion state with the asymptotic solution for the electromagnetic vector potential. In the background magnetic field, the axion-photon interaction induces a mixing, which is parametrized by $\Delta_{B}(r)=\left(g_{\text {ary }} / 2\right) B(r) \sin \Theta$, where $B(r)$ is the strength of the magnetic field at radius $r$ and $\Theta$ is the angle between the radial propagation direction and the magnetic field, which is $r$ independent. The term $\Delta_{a}=-m_{a}^{2} /(2 E)$ incorporates the axion mass and is responsible for the slightly different momenta between the axion and photon states. Strong-field QED effects in vacuum give rise to the term $\Delta_{\|}(r)=$ $(7 / 2) E \xi(r) \sin ^{2} \Theta$, with $\xi(r)=\left(\alpha_{\mathrm{em}} / 45 \pi\right)\left[B(r) / B_{\text {crit }}\right]^{2}$, with $B_{\text {crit }}=m_{e}^{2} / e \approx 4.41 \times 10^{13} \mathrm{G}$ the critical field strength. In general, $\Delta_{\|}$also contains a term related to the photon's effective plasma mass $\omega_{\mathrm{pl}}, \Delta_{\|}=-\omega_{\mathrm{pl}}^{2} /(2 E)$, but this term is subdominant to the QED one for the systems that we consider.

Since the $B$ field strength decreases as we move farther away from the surface of the MWD, it is important to solve the equation of motion Eq. (2) including the changing magnetic field profile with $r$. In the weak-mixing limit we can solve Eq. (2) using the formalism of time-dependent perturbation theory, and the axion-photon conversion probability is found to be $[16,23]$

$$
p_{a \rightarrow \gamma}=\left|\int_{R_{\mathrm{WD}}}^{\infty} d r^{\prime} \Delta_{B}\left(r^{\prime}\right) e^{i \Delta_{a} r^{\prime}-i \int_{R_{\mathrm{WD}}}^{r^{\prime}} d r^{\prime \prime} \Delta_{\|}\left(r^{\prime \prime}\right)}\right|^{2} .
$$

The integral starts at the star's surface, $r=R_{\mathrm{WD}}$, since it is assumed that $\mathrm{x}$-ray photons produced inside the MWD cannot escape. To first approximation we may model the magnetic field outside of the MWD as a magnetic dipole, such that $B(r)=\left(R_{\mathrm{WD}} / r\right)^{3} B_{0}$, with $B_{0}$ the field at the surface and $r>R_{\mathrm{WD}}$. In the SM [59] we consider more general magnetic field configurations, but the results are largely the same. In general, we evaluate Eq. (3) numerically. Note that for typical MWD parameters and asymptotically small $m_{a}$, the conversion probabilities are of

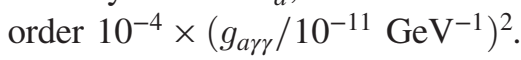

Having calculated both the spectrum of axion radiation $d L_{a} / d E$ and the axion-to-photon conversion probability $p_{a \rightarrow \gamma}$, the flux of axion-induced x-ray photons at Earth is calculated as

$$
\frac{d F_{\gamma_{a}}}{d E}(E)=\frac{d L_{a}}{d E}(E) p_{a \rightarrow \gamma}(E) \frac{1}{4 \pi d_{\mathrm{WD}}^{2}},
$$

where $d_{\mathrm{WD}}$ is the distance between Earth and the MWD. Note that for a typical MWD $d_{\mathrm{WD}} \approx 30 \mathrm{pc}$ away, combining Eq. (4) with the conversion probability estimate and the axion luminosity Eq. (1) leads to axioninduced x-ray fluxes of order $F_{\gamma a} \sim 10^{-15} \times\left(g_{a e e} g_{a \gamma \gamma} /\right.$ $\left.10^{-24} \mathrm{GeV}^{-1}\right)^{2} \mathrm{erg} \mathrm{cm}^{-2} \mathrm{~s}^{-1}$ at low axion masses, though in practice we compute $d F_{\gamma_{a}} / d E$ precisely using the above formalism given the properties of the MWD under consideration. In practice we compute the integrated flux $F_{2-10}$, defined as the integral of Eq. (4) from 2 to $10 \mathrm{keV}$, to compare to data.

The only parameter that appears in Eq. (4) that cannot be measured directly for a given MWD is the core temperature $T_{c}$, which affects the axion luminosity through Eq. (1). The core temperature is not directly observable, since the thin WD atmosphere is largely opaque to radiation. The effective temperature $T_{\text {eff }}$ of the atmosphere, which determines the observed luminosity $L_{\gamma}$, is much smaller than the temperature of the isothermal core $T_{c}$. Understanding the relation between $L_{\gamma}$ and $T_{c}$ requires detailed modeling of the layers connecting the atmosphere to the degenerate core (see, e.g., Refs. [14,65-67]). We use the result of models presented in Ref. [14], which finds that for luminosities above $L_{\gamma} \sim 10^{-3.8} L_{\odot}$ and below $\sim 10^{-1.5} L_{\odot}$ the luminositycore temperature relation is well approximated by

$$
T_{c} \simeq\left(3 \times 10^{6} \mathrm{~K}\right)\left(\frac{L_{\gamma}}{10^{-4} L_{\odot}}\right)^{0.4} .
$$

As we discuss further in the SM, the WD models in Refs. [14,65-67] agree to within $O(10 \%)$ uncertainty [59].

It is important to note that while the effective temperatures $T_{\text {eff }}$ are often at the level of $10^{4} \mathrm{~K}$, the emission from the WD does not follow a pure thermal distribution at this temperature since higher frequencies probe deeper within the WD atmosphere [68]. Still, for the hot WDs we consider, the expected thermal hard x-ray flux (e.g., from 2 to $10 \mathrm{keV}$ ) is negligible compared to the axion-induced fluxes that will be probed.

$X$-ray data analysis and projections.-Although there are over 70000 known WD stars within approximately $100 \mathrm{pc}$ of Earth [69], the total number of known MWDs with well-measured properties is only around 200 [13]. In the SM we list several MWDs that are expected to have the largest axion-induced x-ray flux [59], while in this section we focus on the most promising candidate.

The magnetic WD RE J0317-853 [70] is especially hot, has a strong magnetic field, and is relatively nearby, making it an excellent target for $\mathrm{x}$-ray searches for axions. Optical and UV observations suggest an effective temperature between $T_{\text {eff }}=30000$ and $60000 \mathrm{~K}$, while at the same time being incredibly massive, $M_{\mathrm{WD}} \sim 1.3 M_{\odot}$, and compact, $R_{\mathrm{WD}} \sim$ $0.003-0.004 R_{\odot}[70,71]$. Note that the best-fit temperature presented in Ref. [70] is $T_{\text {eff }}=4.93_{-0.12}^{+0.22} \times 10^{4} \mathrm{~K}$, though a wider range was considered in Ref. [71]. The MWD rotates with a period of $\sim 725 \mathrm{~s}$, and over this period the surface magnetic field strength is seen to oscillate. Reference [72] used time-resolved far-UV spectroscopic data from the Hubble Space Telescope to study the variations in the locations and magnitudes of Lyman $\alpha$ transition to measure the magnetic field profile. They found that the observable surface magnetic field varies between 200 and 800 MG 
over the WD rotation period, which is well fit by an offset dipole model [72]. They also find a best-fit temperature $T_{\text {eff }} \sim 40000 \mathrm{~K}$.

In the SM we model in detail the magnetic field structure of this MWD and assess the sensitivity of our results to uncertainties in the value of $T_{\text {eff }}$, for example [59]. Here, however, we simply calculate the predicted x-ray flux using Eq. (3) with the most conservative choice of parameters for the magnetic field strength and effective temperature of RE J0317-853. We use a central dipole field with surface-field value $B_{0}=200 \mathrm{MG}$ and $\sin \Theta=1$, corresponding to the lowest surface-field value observed in Ref. [72] anywhere on the WD surface over its period. We also take the fiducial values $T_{\text {eff }}=30000 \mathrm{~K}, M_{\mathrm{WD}}=1.32 M_{\odot}$, and $R_{\mathrm{WD}}=$ $0.00405 R_{\odot}$ [71], which let us infer $L_{\gamma}=0.0120 L_{\odot}$ from the Stefan-Boltzmann law and $T_{c}=2.0 \times 10^{7} \mathrm{~K}$ from Eq. (5). We note that higher $T_{\text {eff }}$ lead to higher flux rates, and our fiducial value for $T_{\text {eff }}$ is the lowest end of the range discussed in Refs. [70,71]. Parallax measurements from Gaia-DR2 [69] place RE J0317-853 at a distance of $d_{\mathrm{WD}}=$ $29.54 \pm 0.04$ pc from Earth.

The axion-induced x-ray spectrum from RE J0317-853 should peak around $E \sim 3 T_{c} \sim 5 \mathrm{keV}$. Observations with Suzaku, using approximately $60 \mathrm{ks}$ of exposure time, detected no astrophysical $x$-ray emission from this MWD and set a flux limit in the 2-10 keV range of $F_{2-10}<$ $1.7 \times 10^{-13} \mathrm{erg} \mathrm{cm}^{-2} \mathrm{~s}^{-1}$ at $95 \%$ confidence [73]. We note that the limit in Ref. [73] required background subtraction and modeling; the limit itself is dominated by systematic uncertainties in modeling the cosmic $\mathrm{x}$-ray background and the non-x-ray background. We also caution that the limit in Ref. [73] is formally only valid for an energy spectrum that resembles the above backgrounds, given the energy dependence of the Suzaku effective area, though we have checked that this only induces a $\sim 10 \%$ difference in the predicted counts and thus can be ignored for our purposes. We translate the flux limit into a 95\% constraint on the axion coupling constants $\left|g_{\text {ary }} g_{\text {aee }}\right|$ using the fiducial values for RE J0317853 and the formalism for axion-photon conversion developed above. Our results are presented in Fig. 2, which shows our constraint on the axion parameter space; the region above the blue curve is excluded at $95 \%$ confidence for the fiducial stellar parameters ( $T_{\text {eff }}, B_{0}$, etc.) given above. Note that the nontrivial structure in the limit at high $m_{a}$ is due to transitioning across the regime where $\Delta_{a} \ll \Delta_{\|}$and then again to the regime where $\Delta_{a} \gg R_{\mathrm{WD}}$.

We compare our result to the previous best limits on this coupling combination. By searching for axions produced inside of the Sun though the axion-electron coupling, the CAST experiment obtains a direct limit on $\left|g_{\text {arr }} g_{\text {aee }}\right|$ [74]. Our constraints are approximately 2 orders of magnitude stronger than those from CAST at low axion masses. Several experiments derive upper limits on $\left|g_{\text {ary }}\right|$ alone. CAST provides an upper limit on $\left|g_{a \gamma \gamma}\right|$ from axions produced in the Sun through the Primakoff process [26]. Similarly, an

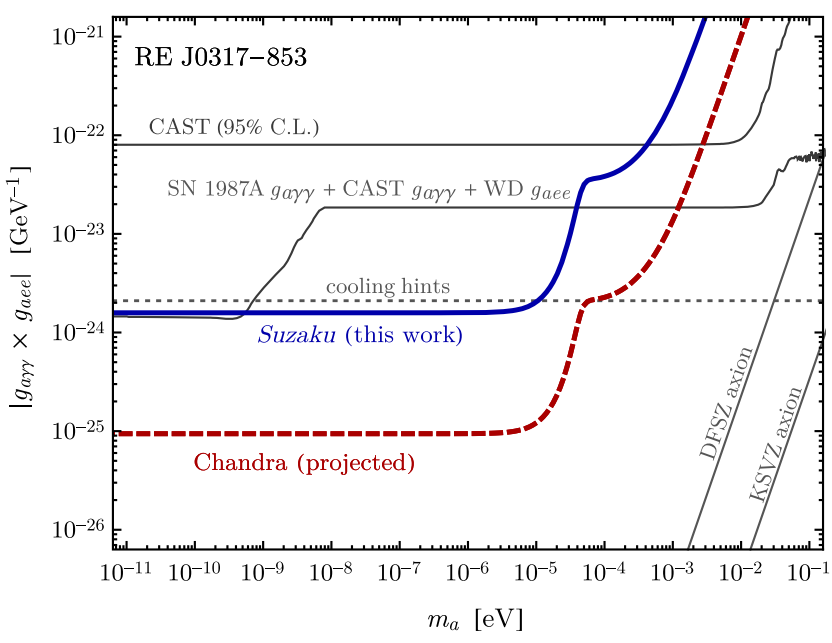

FIG. 2. The $95 \%$ C.L. upper limit on the axion couplings $\left|g_{\text {ary }} g_{\text {aee }}\right|$ as a function of the axion mass $m_{a}$ are inferred from the nonobservation of x-ray emission from the MWD RE J0317-853 by the $S u z a k u$ telescope with $\sim 60 \mathrm{ks}$ of exposure (solid blue line). We also show the $95 \%$ C.L. projected sensitivity from Chandra observations of the same MWD with a $400 \mathrm{ks}$ exposure (dashed red line). The limits extend to $m_{a}=0$ outside of the plotted range. The nontrivial structure in the limit at high $m_{a}$ arises from the transition probability becoming suppressed in this regime by the axion-photon momentum mismatch (see text for details). Additionally, we show the strongest upper limit on this parameter space before this work from the nonobservation of $\gamma$ rays from SN 1987A, searches for axions with the CAST experiment, and constraints on the WD luminosity function. Stellar cooling hints suggest an axion may be present in the spectrum with $\left|g_{\text {ary }} g_{\text {aee }}\right| \sim$ $2 \times 10^{-24}$, as indicated, though this interpretation is subject to large uncertainties.

upper bound on $\left|g_{a \gamma \gamma}\right|$ is derived from the nonobservation of a $\gamma$-ray flux coincident with SN 1987A [75], which is expected to be seen if axions are produced during the corecollapse supernova and subsequently convert into $\gamma$-ray photons in the intergalactic magnetic field. By combining these limits on $g_{a \gamma \gamma}$ with the limit $\left|g_{a e e}\right|<2.8 \times 10^{-13}$, which arises from modeling the WD luminosity function [10], we obtain a second $95 \%$ confidence upper limit, which is stronger than CAST's direct limit on $\left|g_{\text {ary }} g_{\text {aee }}\right|$.

In Fig. 2 we also show the best-fit value for $g_{a \gamma \gamma} g_{a e e}$ ("cooling hints") found in Ref. [76] from a global fit to the available stellar cooling data, which slightly favors nonzero axion-electron and axion-photon couplings. Unfortunately the global fits say little about the axion mass, since the stellar cooling probes are not sensitive to the mass of the particle causing the cooling. From our work we are able to say that the axion mass at these best-fit couplings needs to be above $\sim 2 \times 10^{-5} \mathrm{eV}$. The coupling-mass relations for the Dine-Fischler-Srednicki-Zhitnitsky [20,21] and KimShifman-Vainshtein-Sakharov [17-19] QCD axion models are also shown in Fig. 2.

The Suzaku observations of MWD RE J0317-853 from Ref. [73] are not ideal for searching for the proposed 
axion-induced x-ray signal. This is because Suzaku has a rather poor point-source sensitivity in the $\sim 2-10 \mathrm{keV}$ band compared to other telescopes like XMM-Newton or Chandra. For instance, we estimate that a $\sim 400$ ks observation with Chandra would yield a 95\% confidence flux sensitivity in the $2-10 \mathrm{keV}$ band at the level of $6 \times 10^{-16} \mathrm{erg} \mathrm{cm}^{-2} \mathrm{~s}^{-1}$ [77], which is over 2 orders of magnitude better than the sensitivity achieved in Ref. [73] with Suzaku. (Specifically, this flux sensitivity estimate includes X-ray and particle backgrounds as estimated in Ref. [77] and is for the ACIS-I instrument in the timed exposure mode with no grating and with CCD I3, along with very faint telemetry.) This projected sensitivity appears in Fig. 2 as the red dashed curve. Note that this sensitivity projection assumes that the MWD does not produce $\mathrm{X}$-ray emission in the energy range of interest at the flux levels that would be probed by XMM-Newton and Chandra. This assumption is justified because the thermal emission is exponentially suppressed in the energy range $2-10 \mathrm{keV}$ and well below the levels that would be probed by future observations. Possible nonthermal emission mechanisms include synchrotron and curvature radiation in the strong magnetic field surrounding the MWD. However, these processes are suppressed by the rather large spin period of the MWD, which leads to a small accelerating potential compared to, e.g., the fast-spinning cataclysmic variable (CV) MWD AE Aquarii, for which pulsed nonthermal emission has been possibly observed [73,78].

Discussion.-In this Letter we used Suzaku observations of the nearby MWD RE J0317-853 to set the strongest limits to date on $\left|g_{\text {ary }} g_{\text {aee }}\right|$ for axion masses $\lesssim \mathrm{meV}$. Dedicated observations, with existing telescopes such as XMM-Newton, Chandra, or NuSTAR of this MWD and others have the potential to improve the sensitivity by over an order of magnitude and perhaps more. If the previously observed stellar cooling hints are due to low-mass axionlike-particles, x-ray observations of MWDs should detect excess hard $\mathrm{x}$-ray flux.

If a hard x-ray signal is seen from a MWD, the first question should be if the MWD is accreting, which would be the case for a CV star. CVs, which often emit hard x-ray spectra, are distinguishable from, e.g., their variability and emission line spectra. It is also possible that some MWDs emit hard $\mathrm{x}$ rays due to chromospheric activity, though this has yet to be observed [79]. Our predicted axion signal has the unique feature of having an order unity modulation fraction over the MWD period for most alignment angle configurations, as discussed more in the SM [59], which could help differentiate it from other backgrounds.

The signal discussed in this work requires the axion to interact with both electromagnetism and electrons. While both interactions are expected in a generic ALP theory, from an effective field theory point of view, this fact makes it hard to directly compare our sensitivity to, e.g., the CAST limits on $g_{a \gamma \gamma}$ alone. However, in the SM [59] we show that even if one assumes that the axion-electron operator is generated through the renormalization group alone due to $W$ and $Z$-boson loops, the constraints from this work may be translated to constraints on $g_{\text {ary }}$ that are comparable to those from CAST.

In addition to the axion-electron coupling, a generic axion is also expected to interact derivatively with quarks. These interactions cause NSs to radiate $\sim \mathrm{keV}$ axions, as in the WD case described in this work. NSs are also promising targets for axion-induced hard x-ray signals, and this will be explored in future work [80].

We are grateful to Raymond Co, Anson Hook, Yoni Kahn, and Nick Rodd for comments on the draft. The work of C. D. and B.R. S. was supported in part by the DOE Early Career Grant No. DE-SC0019225. A. J. L. was supported in part by the DOE under Grant No. DE-SC0007859. This research was supported in part through computational resources and services provided by Advanced Research Computing at the University of Michigan, Ann Arbor.

[1] R. D. Peccei and H. R. Quinn, Phys. Rev. D 16, 1791 (1977).

[2] R. D. Peccei and H. R. Quinn, Phys. Rev. Lett. 38, 1440 (1977).

[3] S. Weinberg, Phys. Rev. Lett. 40, 223 (1978).

[4] F. Wilczek, Phys. Rev. Lett. 40, 279 (1978).

[5] P. Svrcek and E. Witten, J. High Energy Phys. 06 (2006) 051.

[6] A. Arvanitaki, S. Dimopoulos, S. Dubovsky, N. Kaloper, and J. March-Russell, Phys. Rev. D 81, 123530 (2010).

[7] G. G. Raffelt, Phys. Lett. 166B, 402 (1986).

[8] J. Isern, E. Garcia-Berro, S. Torres, and S. Catalan, Astrophys. J. 682, L109 (2008).

[9] J. Isern, S. Catalan, E. Garcia-Berro, and S. Torres, J. Phys. Conf. Ser. 172, 012005 (2009).

[10] M. M. Miller Bertolami, B. E. Melendez, L. G. Althaus, and J. Isern, J. Cosmol. Astropart. Phys. 10 (2014) 069.

[11] J. Isern, E. Garcia-Berro, L. G. Althaus, and A. H. Corsico, Astron. Astrophys. 512, A86 (2010).

[12] S. O. Kepler, I. Pelisoli, S. Jordan, S. J. Kleinman, D. Koester, B. Külebi, V. Peçanha, B. G. Castanheira, A. Nitta, J. E. S. Costa, D. E. Winget, A. Kanaan, and L. Fraga, Mon. Not. R. Astron. Soc. 429, 2934 (2013).

[13] L. Ferrario, D. de Martino, and B. Gaensicke, Space Sci. Rev. 191, 111 (2015).

[14] G. Chabrier, P. Brassard, G. Fontaine, and D. Saumon, Astrophys. J. 543, 216 (2000).

[15] D. E. Morris, Phys. Rev. D 34, 843 (1986).

[16] G. Raffelt and L. Stodolsky, Phys. Rev. D 37, 1237 (1988).

[17] M. Dine, W. Fischler, and M. Srednicki, Phys. Lett. 104B, 199 (1981).

[18] A. R. Zhitnitsky, Yad. Fiz. 31, 497 (1980) [Sov. J. Nucl. Phys. 31, 260 (1980)].

[19] M. Srednicki, Nucl. Phys. B260, 689 (1985).

[20] J. E. Kim, Phys. Rev. Lett. 43, 103 (1979).

[21] M. A. Shifman, A. I. Vainshtein, and V. I. Zakharov, Nucl. Phys. B166, 493 (1980).

[22] J.-F. Fortin and K. Sinha, J. High Energy Phys. 06 (2018) 048. 
[23] J.-F. Fortin and K. Sinha, J. High Energy Phys. 01 (2019) 163.

[24] M. Arik et al. (CAST Collaboration), Phys. Rev. Lett. 112, 091302 (2014).

[25] M. Arik et al. (CAST Collaboration), Phys. Rev. D 92 , 021101 (2015).

[26] V. Anastassopoulos et al. (CAST Collaboration), Nat. Phys. 13, 584 (2017).

[27] K. Zioutas, M. Tsagri, Y. Semertzidis, T. Papaevangelou, T. Dafni, and V. Anastassopoulos, New J. Phys. 11, 105020 (2009).

[28] M. S. Pshirkov and S. B. Popov, J. Exp. Theor. Phys. 108, 384 (2009).

[29] F. P. Huang, K. Kadota, T. Sekiguchi, and H. Tashiro, Phys. Rev. D 97, 123001 (2018).

[30] A. Hook, Y. Kahn, B. R. Safdi, and Z. Sun, Phys. Rev. Lett. 121, 241102 (2018)

[31] B. R. Safdi, Z. Sun, and A. Y. Chen, Phys. Rev. D 99, 123021 (2019).

[32] A. Caputo, M. Regis, M. Taoso, and S. J. Witte, J. Cosmol. Astropart. Phys. 03 (2019) 027

[33] G. G. Raffelt, Lect. Notes Phys. 741, 51 (2008).

[34] T. Fischer, S. Chakraborty, M. Giannotti, A. Mirizzi, A. Payez, and A. Ringwald, Phys. Rev. D 94, 085012 (2016).

[35] A. Ayala, I. Domínguez, M. Giannotti, A. Mirizzi, and O. Straniero, Phys. Rev. Lett. 113, 191302 (2014).

[36] M. Simet, D. Hooper, and P. D. Serpico, Phys. Rev. D 77, 063001 (2008).

[37] M. A. Sánchez-Conde, D. Paneque, E. Bloom, F. Prada, and A. Domínguez, Phys. Rev. D 79, 123511 (2009).

[38] A. De Angelis, G. Galanti, and M. Roncadelli, Phys. Rev. D 84, 105030 (2011); 87, 109903(E) (2013).

[39] M. Ajello et al. (Fermi-LAT Collaboration), Phys. Rev. Lett. 116, 161101 (2016).

[40] M. Battaglieri et al., arXiv:1707.04591.

[41] T. M. Shokair et al., Int. J. Mod. Phys. A 29, 1443004 (2014).

[42] N. Du et al. (ADMX Collaboration), Phys. Rev. Lett. 120, 151301 (2018).

[43] B. M. Brubaker, L. Zhong, Y. V. Gurevich, S. B. Cahn, S. K. Lamoreaux, M. Simanovskaia, J. R. Root, S. M. Lewis, S. Al Kenany, K. M. Backes et al., Phys. Rev. Lett. 118, 061302 (2017).

[44] S. Al Kenany et al., Nucl. Instrum. Methods A 854, 11 (2017).

[45] B. M. Brubaker, L. Zhong, S. K. Lamoreaux, K. W. Lehnert, and K. A. van Bibber, Phys. Rev. D 96, 123008 (2017).

[46] A. Caldwell, G. Dvali, B. Majorovits, A. Millar, G. Raffelt, J. Redondo, O. Reimann, F. Simon, and F. Steffen (MADMAX Working Group), Phys. Rev. Lett. 118, 091801 (2017).

[47] Y. Kahn, B. R. Safdi, and J. Thaler, Phys. Rev. Lett. 117, 141801 (2016).

[48] J. W. Foster, N. L. Rodd, and B. R. Safdi, Phys. Rev. D 97, 123006 (2018).

[49] J. L. Ouellet et al., Phys. Rev. Lett. 122, 121802 (2019).

[50] S. Chaudhuri, P. W. Graham, K. Irwin, J. Mardon, S. Rajendran, and Y. Zhao, Phys. Rev. D 92, 075012 (2015).

[51] M. Silva-Feaver et al., IEEE Trans. Appl. Supercond. 27, 1400204 (2016).

[52] D. Budker, P. W. Graham, M. Ledbetter, S. Rajendran, and A. O. Sushkov, Phys. Rev. X 4, 021030 (2014).
[53] R. Bähre et al., J. Instrum. 8, T09001 (2013).

[54] Z. Bogorad, A. Hook, Y. Kahn, and Y. Soreq, Phys. Rev. Lett. 123, 021801 (2019).

[55] M. Tanabashi et al. (Particle Data Group), Phys. Rev. D 98 , 030001 (2018).

[56] M. Nakagawa, T. Adachi, Y. Kohyama, and N. Itoh, Astrophys. J. 326, 241 (1988).

[57] M. Nakagawa, Y. Kohyama, and N. Itoh, Astrophys. J. 322, 291 (1987).

[58] G. G. Raffelt, Phys. Rep. 198, 1 (1990).

[59] See Supplemental Material at http://link.aps.org/ supplemental/10.1103/PhysRevLett.123.061104, where we derive and numerically solve the relevant equations of motion for the more general trajectories and field configurations appropriate for a realistic MWD with finite extent, but the results are similar to those found by assuming radial trajectories. The Supplemental Material includes Refs. [60-64]

[60] S. J. Kleinman et al., Astrophys. J. Suppl. Ser. 204, 5 (2013)

[61] M. Bauer, M. Neubert, and A. Thamm, J. High Energy Phys. 12 (2017) 044.

[62] D. Horns and M. Meyer, J. Cosmol. Astropart. Phys. 02 (2012) 033.

[63] M. Meyer, D. Horns, and M. Raue, Phys. Rev. D 87, 035027 (2013).

[64] A. Abramowski et al. (H.E.S.S. Collaboration), Phys. Rev. D 88, 102003 (2013).

[65] B. M. S. Hansen, Astrophys. J. 520, 680 (1999).

[66] M. Salaris, E. Garcia-Berro, M. Hernanz, J. Isern, and D. Saumon, Astrophys. J. 544, 1036 (2000).

[67] I. Renedo, L. G. Althaus, M. M. M. Bertolami, A. D. Romero, A. H. Corsico, R. D. Rohrmann, and E. GarciaBerro, Astrophys. J. 717, 183 (2010).

[68] H. L. Shipman, Astrophys. J. Lett. 206, L67 (1976).

[69] A. G. A. Brown et al. (Gaia Collaboration), Astron. Astrophys. 616, A1 (2018).

[70] M. A. Barstow, S. Jordan, D. O'Donoghue, M. R. Burleigh, R. Napiwotzki, and M. K. Harrop-Allin, Mon. Not. R. Astron. Soc. 277, 971 (1995).

[71] B. Külebi, S. Jordan, E. Nelan, U. Bastian, and M. Altmann, Astron. Astrophys. 524, A36 (2010).

[72] M. R. Burleigh, S. Jordan, and W. Schweizer, Astrophys. J. 510, L37 (1999).

[73] A. Harayama, Y. Terada, M. Ishida, T. Hayashi, A. Bamba, and M. S. Tashiro, Publ. Astron. Soc. Jpn. 65, 73 (2013).

[74] K. Barth et al., J. Cosmol. Astropart. Phys. 05 (2013) 010.

[75] A. Payez, C. Evoli, T. Fischer, M. Giannotti, A. Mirizzi, and A. Ringwald, J. Cosmol. Astropart. Phys. 02 (2015) 006.

[76] M. Giannotti, I. G. Irastorza, J. Redondo, A. Ringwald, and K. Saikawa, J. Cosmol. Astropart. Phys. 10 (2017) 010.

[77] Chandra X-Ray Observatory, Chandra Science Tools, 2019, http://cxc.harvard.edu/toolkit/pimms.jsp.

[78] Y. Terada, T. Hayashi, M. Ishida, K. Mukai, T. u. Dotani, S. Okada, R. Nakamura, S. Naik, A. Bamba, and K. Makishima, Publ. Astron. Soc. Jpn. 60, 387 (2008).

[79] C. S. Brinkworth, M. R. Burleigh, G. A. Wynn, and T. R. Marsh, Mon. Not. R. Astron. Soc. 348, L33 (2004).

[80] R. T. Co, C. Dessert, Y. Kahn, and B. R. Safdi (to be published) 